\title{
A Human Head and Neck Squamous Cell Carcinoma Cell Line with Acquired cis-Diamminedichloroplatinum-Resistance Shows Remarkable Upregulation of BRCA1 and Hypersensitivity to Taxane
}

\author{
Yuriko Saiki, ${ }^{1}$ Takenori Ogawa, ${ }^{1,2}$ Kiyoto Shiga, ${ }^{2}$ Makoto Sunamura, ${ }^{1,3}$ \\ Toshimitsu Kobayashi, ${ }^{2}$ and Akira Horii ${ }^{1}$ \\ ${ }^{1}$ Department of Molecular Pathology, Tohoku University School of Medicine, Sendai, Miyagi 980-8575, Japan \\ ${ }^{2}$ Department of Otolaryngology-Head and Neck Surgery, Tohoku University School of Medicine, Sendai, Miyagi 980-8574, Japan \\ ${ }^{3}$ Department of Digestive Tract Surgery and Transplantation Surgery, Tokyo Medical University Hachioji Medical Center, Hachioji, \\ Tokyo 193-0998, Japan
}

Correspondence should be addressed to Akira Horii, horii@med.tohoku.ac.jp

Received 7 July 2011; Accepted 20 August 2011

Academic Editor: Leonard P. Rybak

Copyright ( 2011 Yuriko Saiki et al. This is an open access article distributed under the Creative Commons Attribution License, which permits unrestricted use, distribution, and reproduction in any medium, provided the original work is properly cited.

\begin{abstract}
Recently, an inverse relationship between resistance to platinum-based chemotherapeutic agents and taxanes has been implicated in breast and ovarian cancers, and a possible pivotal role for BRCA1 has also been suggested. Because cisdiamminedichloroplatinum (CDDP) and taxanes are the most active antitumor agents against head and neck squamous cell carcinoma (HNSCC), we analyzed the sensitivity of nine HNSCC cell lines and their previously established derived CDDP-resistant cell lines to two representative taxanes: docetaxel and paclitaxel. None of the nine original cell lines showed any cross resistance between CDDP and taxanes, but one of the CDDP-resistant cell lines, RPMI2650CR, demonstrated hypersensitivity to both taxanes when compared to the parental cell line, RPMI2650. Furthermore, RPMI2650CR exhibited increased expression of BRCA1. These data suggest that (i) taxanes are a good candidate for a second-line therapeutic drug for HNSCC patients with acquired CDDP resistance and (ii) BRCA1 can be a candidate marker for predicting an inverse CDDP/taxane sensitivity phenotype in HNSCC.
\end{abstract}

\section{Introduction}

Cisplatin (cis-diamminedichloroplatinum, CDDP), a DNA damaging drug, is the most active antitumor agent for treating patients with head and neck squamous cell carcinoma (HNSCC). However, acquired resistance to CDDP is one of the major problems in the clinical management of such patients [1]. CDDP-resistance has been reported to be associated with decreased cellular accumulation of the drug, increased levels of glutathione, DNA repair, and antiapoptotic activity [2]. Hence we focused on factors or molecules that are closely associated with sensitivity and/or resistance to CDDP, and we identified IGF2 as one of the candidate molecules for acquired CDDP resistance [3]. Ultimately, we need to find a way to deduce the molecular mechanisms for acquisition of resistance. In the meantime, it is also very important to find alternative $\operatorname{drug}(\mathrm{s})$ or method(s) for defeating the acquired resistance. Stordal et al. highlighted an inverse relationship between CDDP and taxane resistance [4]. These drugs function in different phases of the cell cycle; thus CDDP and taxanes are often applied as combined chemotherapy [5, 6]. Herein, we studied the inverse relationships of resistance between CDDP and taxanes in HNSCC cell lines and observed that one of our cell lines with acquired CDDP resistance showed increased taxane sensitivity when compared with its parental cell line. Furthermore, dramatic upregulation of BRCA1 was also observed in these CDDPresistant cells. Although our findings are just the tip of the iceberg and many other mechanisms are yet-to-bediscovered, it is possible that the BRCA1 status can be used 
as one of the biomarkers to forejudge the taxane sensitivity of HNSCC with acquired CDDP resistance.

\section{Materials and Methods}

2.1. Cell Lines. Nine human HNSCC cell lines were used; the origins of the cell lines are one nasal cavity cancer (RPMI2650), two maxillary cancers (HSQ89, IMC4), and six oral-cavity cancers (HSC2, HSC3, HSC4, Ca9-22, HO-1-u1, SAS). These cells were grown by methods indicated by the original developers. We also established their derived CDDPresistant (CR) cell lines by a method described elsewhere [7].

2.2. MTT Assay. A total of $5 \times 10^{3}$ cells/well was seeded in each well of flat-bottomed 96-well plates in triplicate and cultured in $200 \mu \mathrm{L}$ of medium. After 18 hours of incubation, either CDDP, or paclitaxel or docetaxel were added to the media using a fivefold dilution series, and the cells were cultured for 48 hours. Then the medium was replaced with $100 \mu \mathrm{L}$ of $0.05 \%$ 3-[4,5-dimethylthiazol-2-yl]-2,5-diphenyltetrazo-lium bromide (MTT)/PBS and incubated for one hour. After the incubation, the MTT solution was removed, and the cells were suspended in 100\% ethanol. Absorption was measured at $590 \mathrm{~nm}$ using a Versamax microplate reader (Amersham Biosciences Corp., Piscataway, NJ). Independent triplicate assays were performed. All values represent the mean \pm standard deviation (SD) from 3 sets of independent cultures.

2.3. Western Blotting Analysis for BRCA1. A total of $2 \times 10^{5}$ cells were harvested, and protein concentrations in total cell lysates were measured using a DC protein assay kit (Bio-Rad, Hercules, CA). A $40 \mu \mathrm{g}$ aliquot of the protein was subjected to immunoblotting using a $10-20 \%$ polyacrylamide gradient gel (Bio-Rad, Hercules, CA). Antibodies used were rabbit anti-BRCA1 monoclonal antibody (Oncogene, Boston, MA), mouse anti- $\beta$ actin monoclonal antibody (Sigma, St Louis, $\mathrm{MO}$ ), and horseradish peroxidase conjugated anti-mouse immunoglobulin antibodies (Amersham Biosciences Corp., Piscataway, NJ). For blocking conditions and concentrations of antibodies, we followed the manufacturer's recommendations. Signals were visualized by reaction with ECL Detection Reagent (Amersham Biosciences Corp., Piscataway, NJ) and digitally processed using LAS 1000 Plus with a Science Lab 99 Image Gauge (Fuji Photo Film, Minamiashigara, Japan).

2.4. Statistical Analysis. The statistical differences in cell viability between the parental and CDDP-resistant cell lines were determined with student's $t$-test, and the significant difference level was established at $P<0.05$.

\section{Results and Discussion}

In a previous study, we successfully established CDDP resistant cell lines from all of the HNSCC cell lines we attempted [3]. These results may indicate that acquisition of CDDP chemoresistance is not a rare event; we frequently observe such acquired resistance in HNSCC patients undergoing

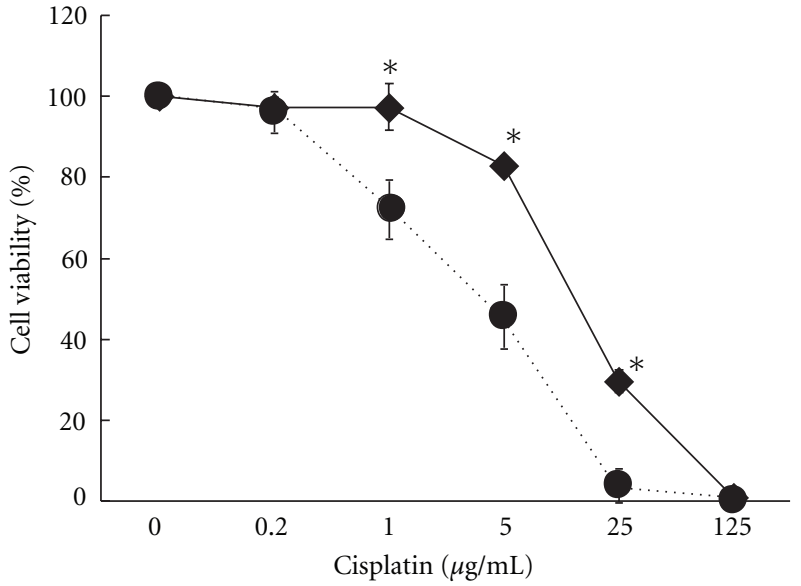

(a)

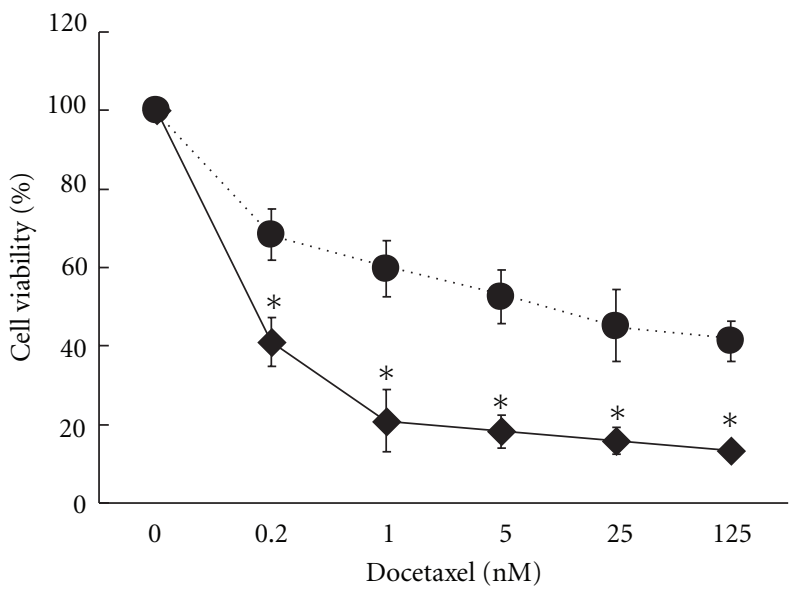

(b)

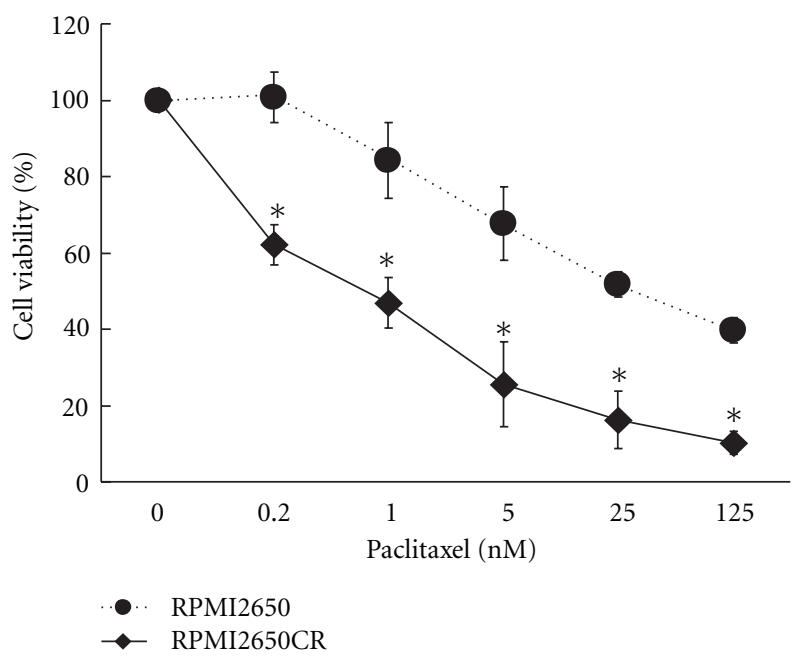

(c)

FIGURE 1: Cell viability with chemotherapeutic agents was assessed by MTT assay. RPMI2650CR shows higher resistance to cisplatin that the parental RPMI2650 (a) but has higher sensitivities to both docetaxel (b) and paclitaxel (c). 


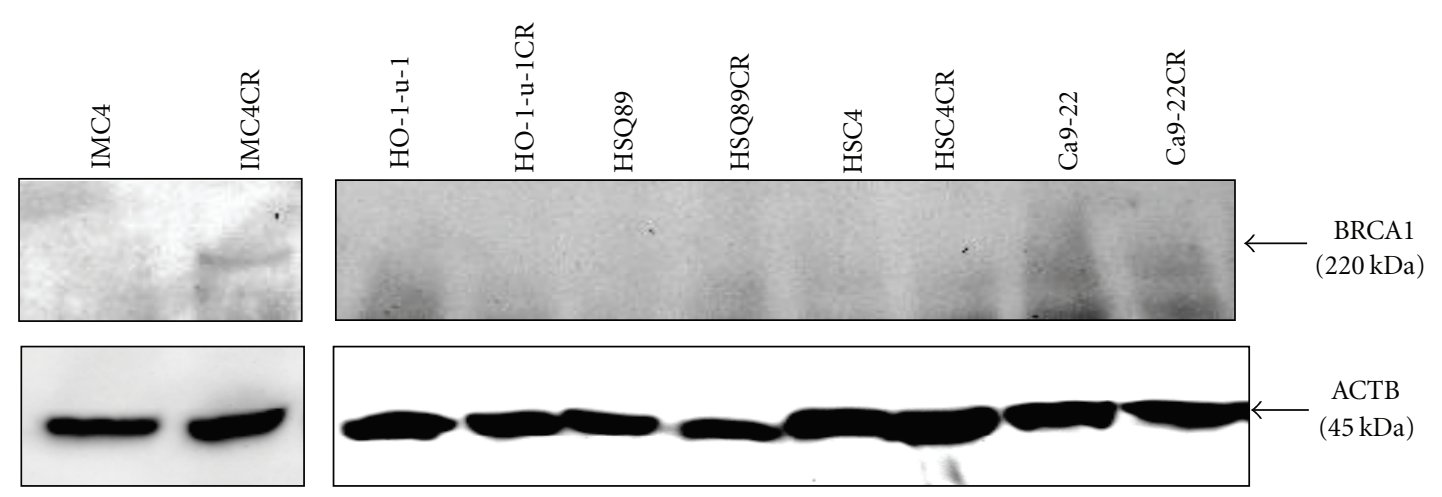

(a)

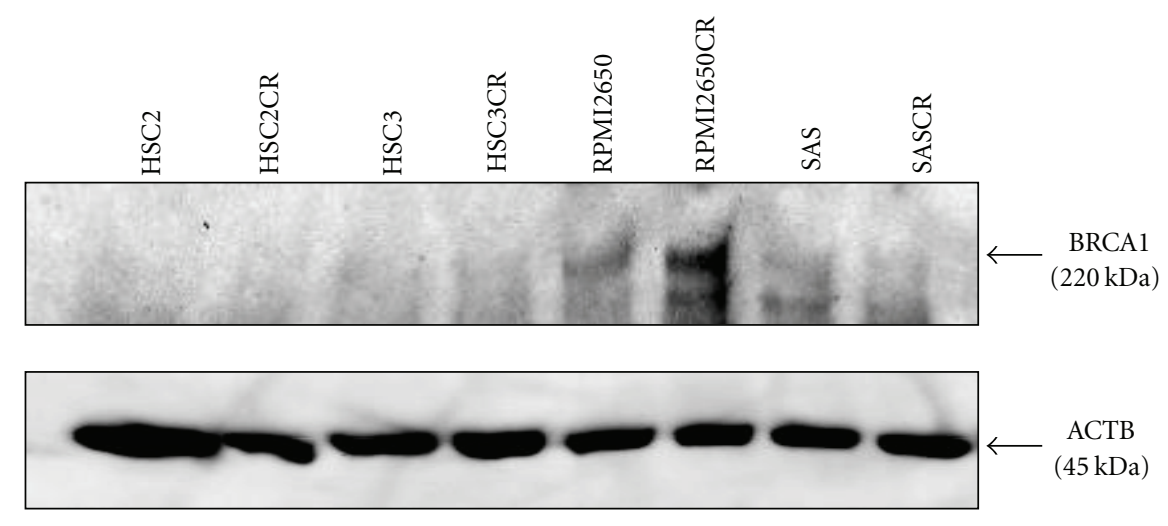

(b)

FIGURE 2: Western blot analysis of BRCA1 in HNSCC cell lines and their derived CDDP-resistant (CR) cell lines. Marked upregulation of BRCA1 $(220 \mathrm{kDa})$ was observed only in the RPMI2650CR cell line. Beta actin (ACTB) was used as an internal control.

chemotherapy in the clinical setting. Hence it is important to understand the mechanisms of acquired resistance to CDDP, as well as trying to find alternative methods for clinical management of patients with acquired CDDP resistance. We also identified IGF2 as one of the candidate genes for acquired CDDP resistance in HNSCC by cDNA microarray analysis, and the stably IGF2-expressing RPMI2650 cells did show resistance to CDDP similar to that observed in RPMI2650CR [3].

Stordal et al. reported an inverse correlation of chemosensitivity between taxane and CDDP [4]. Therefore, we further performed an MTT assay to elucidate taxane sensitivity using our established acquired CDDP-resistant HNSCC cell lines; 8 of the 9 analyzed CDDP-resistant cell lines showed sensitivities similar to their parental cell lines (data not shown), but the RPMI2650-derived CDDP-resistant cell line (RPMI2650CR) demonstrated hypersensitivity to taxanes when compared to the parental cell line (see Figure 1). It is notable that the IC50 value changed by one order of magnitude for CDDP chemosensitivity, but the IC50 values differed by two orders of magnitude in chemosensitivities to both taxanes. These phenomena were reproducible for the two different taxanes. Stably IGF2-expressing RPMI2650 was also used to determine the taxane sensitivity, but it showed a sensitivity similar to that of parental RPMI to both taxanes (data not shown), indicating that IGF2 is not involved in the hypersensitivity to taxanes. Although the molecular mechanisms behind the inverse correlation of chemosensitivity between taxane and CDDP are not well understood, it can be proposed that taxanes are good candidates as a second-line chemotherapeutic choice for HNSCC patients with acquired chemoresistance to CDDP.

Several studies have suggested an association between taxane sensitivity and BRCA1 expression [8], so we then analyzed BRCA1 expression by Western blotting analysis in the 9 acquired CDDP-resistant cell lines we established. Results are shown in Figure 2; prominent upregulation of BRCA1 was observed in RPMI2650CR, and none other cell lines demonstrated a similar pattern. The possible clinical significance of BRCA1 detection is that preoperative immunostaining of a biopsy specimen for BRCA1 may indicate the effectiveness of chemotherapy with taxanes and may possibly additionally estimate CDDP sensitivity, ultimately leading to avoidance of the unnecessary employment of rich CDDP treatment, which has adverse side effects. Further studies are necessary to confirm the association of BRCA1 expression with chemotherapy resistance in HNSCC.

\section{Conclusions}

Acquired CDDP resistance is often observed in patients with HNSCC, and taxanes are used as the second-line chemotherapeutic drug for HNSCC. We observed that an 
acquired CDDP-resistant HNSCC cell line showed higher sensitivity to taxane by two orders of magnitude than its parental cell line. Prominent upregulation of BRCA1 was also observed in this CDDP-resistant/taxane-sensitive cell line. Expression status of BRCA1 can be a biomarker to forejudge the taxane sensitivity to patients with acquired CDDP-resistant HNSCC.

\section{Acknowledgments}

The authors are grateful to Dr. B. L. S. Pierce (University of Maryland University College) for editorial work in the preparation of this paper. This work was supported in part by Grants-in-Aid, by the Academic Frontier Project for Private Universities: matching fund subsidy 2006-2010 from the Ministry of Education, Culture, Sports, Science and Technology of Japan and by a Grant-in-Aid for Cancer Research (Grant no. 18-19) from the Ministry of Health, Labour and Welfare of Japan.

\section{References}

[1] A. A. Forastiere, "Chemotherapy in the treatment of locally advanced head and neck cancer," Journal of Surgical Oncology, vol. 97, no. 8, pp. 701-707, 2008.

[2] L. Kelland, "The resurgence of platinum-based cancer chemotherapy," Nature Reviews Cancer, vol. 7, no. 8, pp. 573-584, 2007.

[3] T. Ogawa, K. Ogawa, K. Shiga et al., "Upregulation of IGF2 is associated with an acquired resistance for cis-diamminedichloroplatinum in human head and neck squamous cell carcinoma," European Archives of Oto-Rhino-Laryngology, vol. 267, no. 10, pp. 1599-1606, 2010.

[4] B. Stordal, N. Pavlakis, and R. Davey, "A systematic review of platinum and taxane resistance from bench to clinic: an inverse relationship," Cancer Treatment Reviews, vol. 33, no. 8, pp. 688703, 2007.

[5] M. R. Posner, D. M. Hershock, C. R. Blajman et al., "Cisplatin and fluorouracil alone or with docetaxel in head and neck cancer," New England Journal of Medicine, vol. 357, no. 17, pp. 1705-1715, 2007.

[6] J. B. Vermorken, E. Remenar, C. Van Herpen et al., "Cisplatin, fluorouracil, and docetaxel in unresectable head and neck cancer," New England Journal of Medicine, vol. 357, no. 17, pp. 1695-1704, 2007.

[7] I. Iwasaki, H. Sugiyama, S. Kanazawa, and H. Hemmi, "Establishment of cisplatin-resistant variants of human neuroblastoma cell lines, TGW and GOTO, and their drug crossresistance profiles," Cancer Chemotherapy and Pharmacology, vol. 49, no. 6, pp. 438-444, 2002.

[8] B. Stordal and R. Davey, "A systematic review of genes involved in the inverse resistance relationship between cisplatin and paclitaxel chemotherapy: role of BRCA1," Current Cancer Drug Targets, vol. 9, no. 3, pp. 354-365, 2009. 


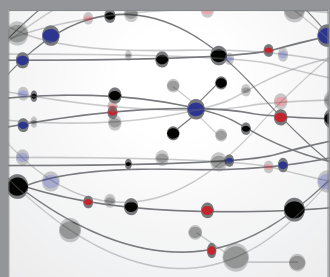

The Scientific World Journal
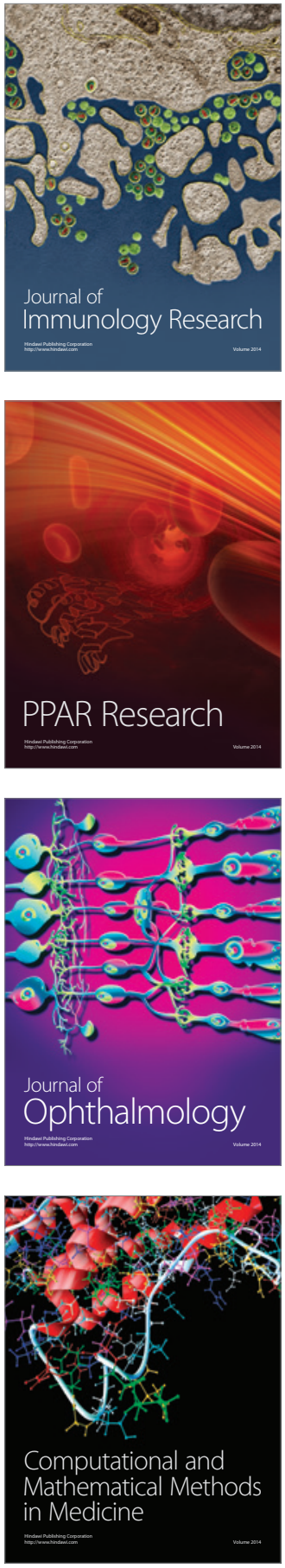

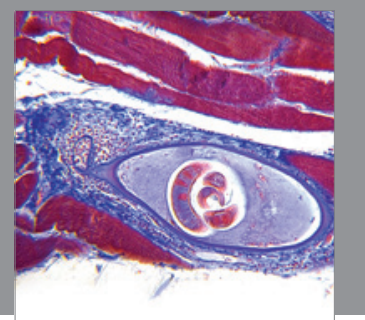

Gastroenterology

Research and Practice
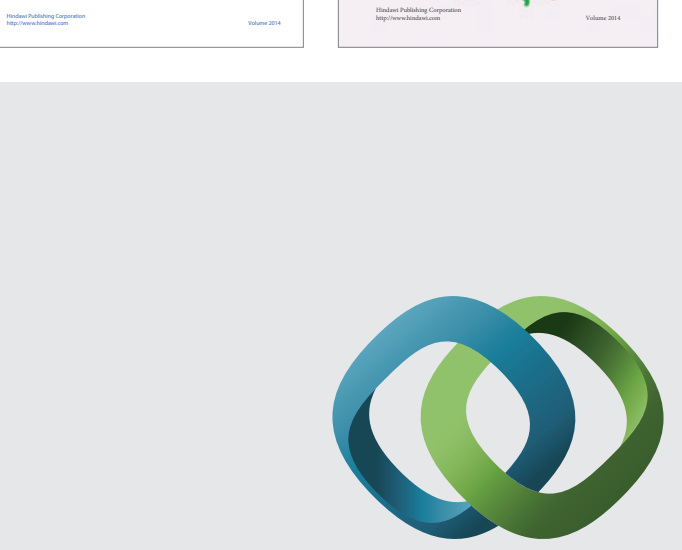

\section{Hindawi}

Submit your manuscripts at

http://www.hindawi.com
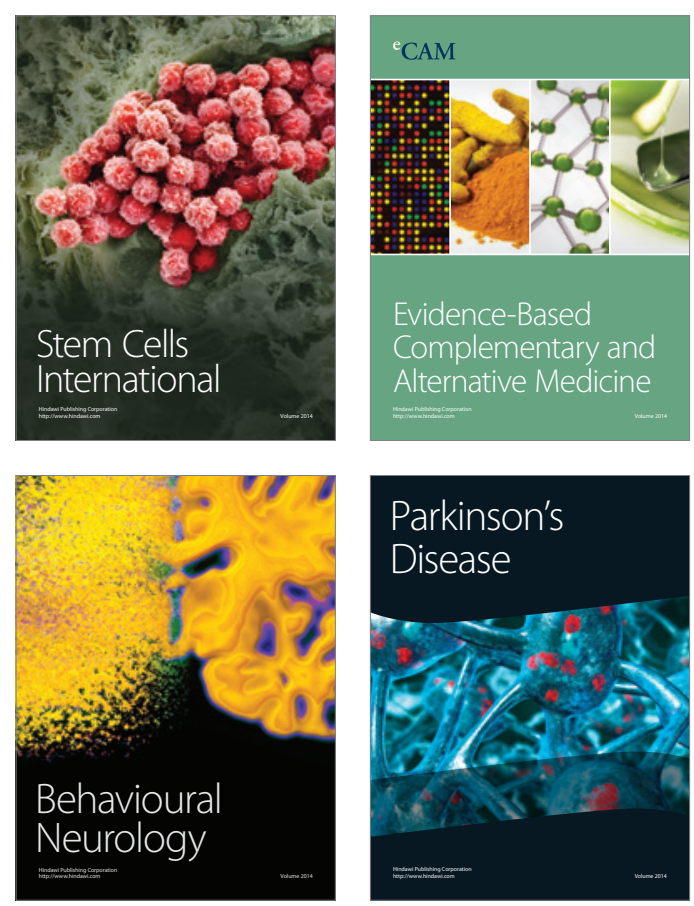

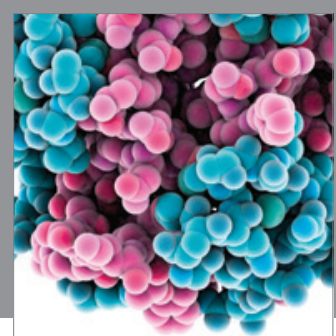

Journal of
Diabetes Research

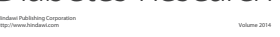

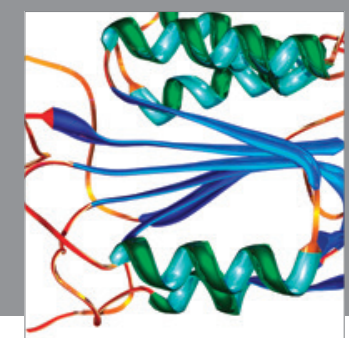

Disease Markers
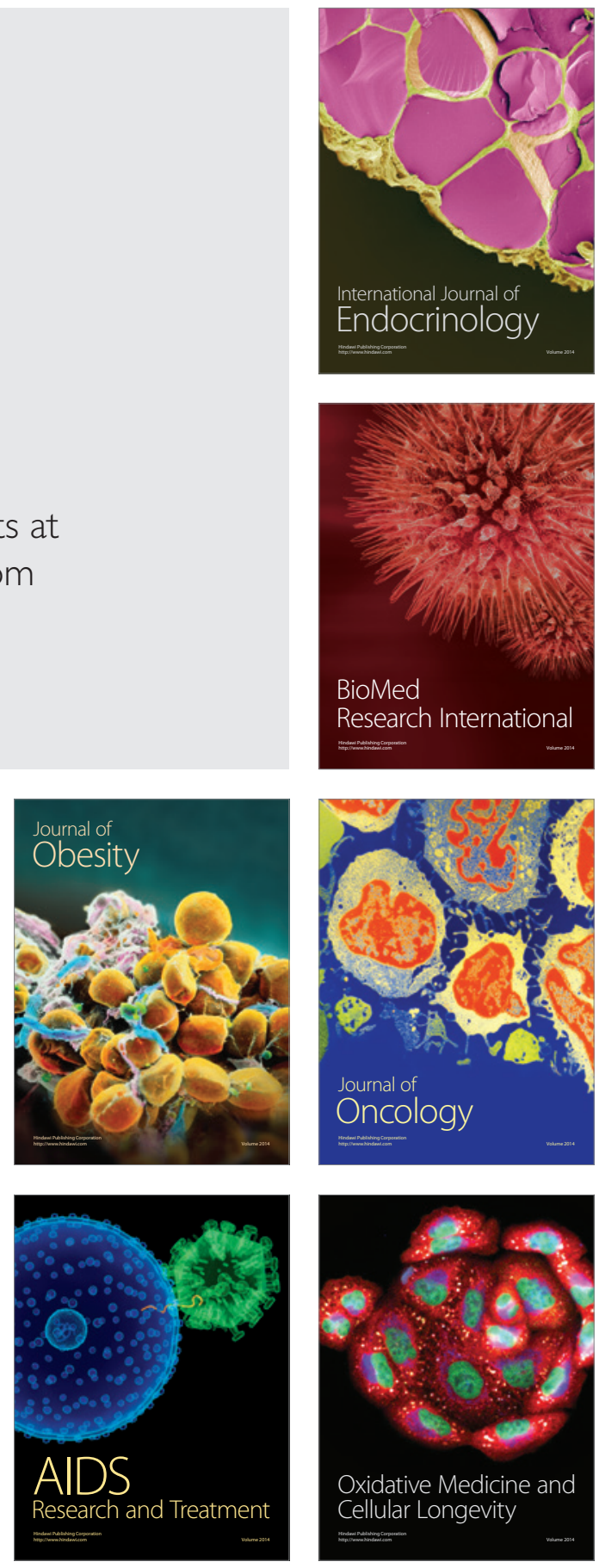